\title{
MODAL ENERGY TRANSFER BY CONTROLLED STRUCTURAL CONNECTIONS
}

\author{
Mariusz OSTROWSKI, Bartlomiej BLACHOWSKI, Lukasz JANKOWSKI, Dominik PISARSKI
}

Institute of Fundamental Technological Research of the Polish Academy of Sciences mostr@ippt.pan.pl; bblach@ippt.pan.pl; ljank@ippt.pan.pl; dpisar@ippt.pan.pl

\section{Abstract}

This paper describes a semi-active control strategy that allows to transfer the vibration energy from an arbitrarily induced to a selected structural mode. The intended aim of the proposed control strategy is energy harvesting from structural vibrations. Another potential application is related to structural safety. In the paper, a mathematical model is first introduced to describe the phenomenon of vibrational energy transfer, and then, based on this model, an efficient semi-active control strategy is proposed. Finally, some problems related to measurement techniques are discussed. The effectiveness of the proposed methodology is demonstrated in an example of energy transfer between vibrational modes of a three-bar planar frame structure.

Keywords: modal control, lockable joint, vibration energy, modal coupling

\section{TRANSFER ENERGII MODALNEJ ZA POMOCĄ STEROWANYCH POŁĄCZEŃ KONSTRUKCYJNYCH}

\section{Streszczenie}

Niniejszy artykuł opisuje półaktywną strategię sterowania umożliwiającą transfer energii z dowolnej wzbudzonej postaci do innej wybranej postaci drgań własnych konstrukcji. Proponowana strategia sterowania może być wykorzystana w celu pozyskiwania energii z drgań konstrukcji. Inne potencjalne zastosowanie związane jest z kwestiami bezpieczeństwa konstrukcji. W artykule najpierw wprowadzony jest model matematyczny opisujący zjawisko transferu energii drgań, a następnie, w oparciu o ten model, przedstawiona jest efektywna strategia sterowania półaktywnego. Na koniec omówiono zagadnienia związane z technikami pomiarowymi. Skuteczność proponowanej metodologii pokazano na przykładzie transferu energii pomiędzy postaciami drgań płaskiej ramy portalowej.

Słowa kluczowe: sterowanie modalne, blokowane węzły, energia drgań, sprzężenie modalne

\section{INTRODUCTION}

In recent years many research papers have been devoted to problems related to semi-active control of structural vibrations. However, a great majority of these papers tackle the problem of vibration mitigation [2], while issues connected with semiactive control strategies for the purpose of energy harvesting are addressed in only a few of them [6, 17].

The purpose of this study is the development of a semi-active control strategy for transferring energy from a randomly induced to an a priori selected modal shape. Such a control strategy is implemented by a set of dynamically activated structural joints, whose behaviour depends on the applied control signals causing their closure or opening. Closed joints are able to transfer bending moments between connected members, while the opened joints behave like pin connections.

The proposed control strategy has many potential applications. Changing the modes of a vibrating structure allows one to modify the amplitudes at the points of the structure at which energy harvesters are located. Lockable joints can be actuated by piezo stacks which are readily available on the market. This type of actuator needs negligibly small amount of energy to lock or unlock the joint, so that the energy which can be potentially harvested from structural vibration is much larger than energy required to control the joint [3].

In addition to the possibility of amplitude maximization at the chosen points of energy harvester location, one can change the frequency along with the vibration mode. It allows to adapt the vibration to energy harvesters that have only one natural frequency, e.g., for autoparametric pendulum damping vibrations with an additionally applied energy harvester shown in [6]

Another interesting application is the transfer of the overall vibrational energy to the selected mode shape, which does not disturb the proper functioning of the structure nor contributes to its fatigue failure. This energy transfer usually does not take more time than one period of the selected vibration 
mode. Such a quick operation of the method is a valuable property in emergency situations.

The proposed control strategy can be classified as modal control. In this case, the control law can be formulated with the use of modal coordinates instead of the physical ones. Such a control with modal filtering based measurement technique is resistant to spillover effects [8]. Examples of applications of semi-active modal control in civil or space structures can be found $[7,13]$.

Compared to fully active control, the semiactive control allows to reduce the control effort of the overall system. What is even more important, it does not cause instability problems [14].

The concept of semi-active control by means of lockable joints has been originally proposed for energy transfer from low to high-frequency modes in order to naturally reduce structural vibrations taking advantage of inherent material damping [10, 15]. Then, this control strategy has been applied for energy transfer to an a priori selected mode in a simple portal frame modelled by 5 DOFs [11]. Recently, it has been used in a frame with a refined finite element discretization [12].

In the present study, a mathematical model of the vibrational energy transfer and the corresponding control law will be proposed. Additionally, a numerical simulation of the energy transfer process in a three-bar frame discretized with 47 DOFs will be demonstrated. It will be shown that, in such a frame, the controlled energy transfer between vibrational modes is possible, even if only a few low-frequency modes are available from measurements.

\section{SYSTEM DYNAMICS AND ENERGY TRANSFER}

\subsection{Dynamics of the structure with lockable joints}

The structure under consideration is discretized with the aid of the finite element (FE) method. It is assumed that a dynamically activated structural joint can operate in two opposite states, namely: locked - with the bending moment being transmitted between the connected structural members, and unlocked - with two independent rotations at the ends of the connected members. Such an assumption allows one to apply a simplified mathematical model of friction in the connection. The completely locked state of the connection is modelled by large values of viscous damping between appropriate rotational degrees of freedom, as shown in Figure 1. In transient analysis, a sufficiently large damping in the connection represents a rigid joint.

Equations of motion for the structural system with lockable joints can be written as follows:

$$
\mathrm{M} \ddot{\mathrm{q}}+\left(\mathrm{C}_{0}+\sum_{k=1}^{N_{k}} u_{k} \mathrm{C}_{k}\right) \dot{\mathrm{q}}+\mathrm{Kq}=\mathrm{f}
$$

where: $\mathrm{q}$ is the response vector in physical coordinates, $\mathrm{M}$ is the mass matrix, $\mathrm{C}_{0}$ is the material damping matrix, $\mathrm{K}$ is the stiffness matrix, $\mathrm{f}$ is the vector of external forces, $N_{k}$ is the total number of the lockable joints, $k$ denotes the index of the lockable joint, $u_{k} \in\{0,1\}$ is the control signal for the $k$ th lockable joint, $\mathrm{C}_{k}$ is the matrix that represents the locked state of the $k$ th controllable joint. Finally, matrix $\mathrm{C}_{k}$ is described by the following formula:

$$
\mathrm{C}_{k}=c_{\mathrm{MAX}} \mathrm{L}_{k}^{\mathrm{T}} \mathrm{L}_{k},
$$

$$
\mathrm{L}_{k}=\left[\begin{array}{lllllllllllll}
0 & \cdots & 0 & 1 & 0 & \cdots & 0 & \cdots & 0 & -1 & 0 & \cdots & 0
\end{array}\right],
$$

where $c_{\text {MAX }}$ is a large viscous damping coefficient. The transformation matrix $\mathrm{L}_{k}$ selects the DOFs that correspond to the $k$ th lockable joint. When the $k$ th lockable joint is currently locked, relative angular velocity $\Delta \dot{q}_{k}$ at the $k$ th joint is suppressed to nearzero value thanks to the large value of the $c_{\operatorname{MAX}}$ coefficient:

$$
\Delta \dot{q}_{k}=\mathrm{L}_{k} \dot{\mathrm{q}} \approx 0 .
$$

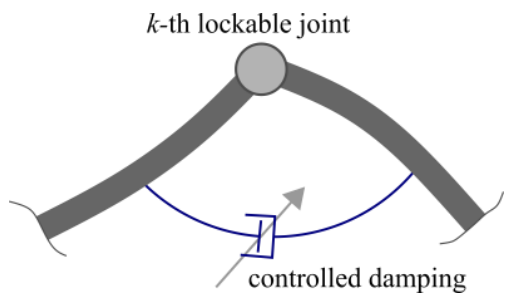

Fig. 1. The concept of lockable joint

\subsection{Effect of modal coupling}

When considering energy transfer in vibrating structures, it is advantageous to perform modal analysis. The uncoupled eigenvalue problem considered in this study takes the following standard form:

$$
\left(\mathrm{K}-\omega_{m}{ }^{2} \mathrm{M}\right) \phi_{m}=0 .
$$

The eigenvector $\phi_{m}$ represents the $m$ th mode shape of the structure with all joints in the unlocked state, i.e., acting as pin connections, $\omega_{m}$ is the $m$ th natural frequency of the system. Additionally, mode shapes are normalized with respect to the mass matrix. After assembling mode shapes into a single matrix $\Phi$, one can formulate the modal transformation as follows:

$$
\mathrm{q}=\Phi \eta,
$$

where $\eta$ is the vector of the newly introduced modal coordinates. Substituting (5) into (1) and leftmultiplying by the transpose of the modal matrix $\Phi^{\mathrm{T}}$, one obtains:

$$
\begin{gathered}
\underbrace{\Phi^{\mathrm{T}} \mathrm{M} \Phi}_{\mathrm{I}} \ddot{\eta}+(\underbrace{\Phi^{\mathrm{T}} \mathrm{C}_{0} \Phi}_{\Gamma_{0}}+\sum_{k=1}^{N_{k}} u_{k} \underbrace{\Phi^{\mathrm{T}} \mathrm{C}_{k} \Phi}_{\Gamma_{k}}) \dot{\eta} \\
+\underbrace{\Phi^{\mathrm{T}} \mathrm{K} \Phi}_{\Omega^{2}} \eta=\Phi^{\mathrm{T}} \mathrm{f} .
\end{gathered}
$$

In eqn. (6) $\mathrm{I}$ is the identity matrix, $\Gamma_{0}$ is the diagonal matrix that contains modal damping coefficients, $\Gamma_{k}$ is the matrix responsible for the coupling of modal equations through the action of the $k$ th lockable 
joint and $\Omega$ is the diagonal matrix of natural frequencies. In this way, the equations of motion (1) are transformed from the configuration space into modal space, which can be written in the matrix notation as

$$
\ddot{\eta}+\left(\Gamma_{0}+\sum_{k=1}^{N_{k}} u_{k} \Gamma_{k}\right) \dot{\eta}+\Omega^{2} \eta=\Phi^{\mathrm{T}} \mathrm{f},
$$

or in the index notation as

$$
\begin{aligned}
\ddot{\eta}_{m}+2 \mu_{m} \omega_{m} \dot{\eta}_{m} & +\sum_{k=1}^{N_{k}} \sum_{n=1}^{N_{m}} u_{k} \gamma_{m n}^{(k)} \dot{\eta}_{n} \\
& +\omega_{m}{ }^{2} \eta_{m}=\sum_{i=1}^{N_{i}} \phi_{m i} f_{i},
\end{aligned}
$$

where $\phi_{m i}$ is the $i$ th component of the $m$ th mode shape $\phi_{m}, i$ is the index related to individual degrees of freedom and $N_{i}$ is the total number of DOFs.

As a consequence of locking the $k$ th joint, the $m$ th modal coordinate becomes coupled with the $n$th mode in the following way:

$$
\begin{aligned}
\gamma_{m n}^{(k)} \dot{\eta}_{n} & =\phi_{m}^{\mathrm{T}} \mathrm{C}_{k} \phi_{n} \dot{\eta}_{n} \\
& =c_{\mathrm{MAX}} \phi_{m}^{\mathrm{T}} \mathrm{L}_{k}^{\mathrm{T}} \mathrm{L}_{k} \phi_{n} \dot{\eta}_{n} \\
& =c_{\mathrm{MAX}} \Delta \phi_{m}^{(k)} \Delta \phi_{n}^{(k)} \dot{\eta}_{n} .
\end{aligned}
$$

$\Delta \phi_{m}^{(k)}$ is the difference of the rotation angles at the $k$ th joint for the $m$ th mode shape, $\Delta \phi_{n}^{(k)} \dot{\eta}_{n}$ is the difference of angular velocities for the $n$th mode shape at the $k$ th joint. The product $\Delta \phi_{m}^{(k)} \Delta \phi_{n}^{(k)}$ in eqn (9) shows that the modal acceleration of the $m$ th mode caused by modal coupling with the $n$th mode is proportional to their local relative rotations of DOFs involved at the $k$ th joint.

From eqn. (9) it follows that the term (8) that couples modal equations can be considered as sum of the modal forces $f_{m k}$ provided by each particular joint. Each $k$ th modal force $f_{m k}$ is as follows:

$$
\begin{aligned}
f_{m k} & =-u_{k} \sum_{n=1}^{N_{m}} \gamma_{m n}^{(k)} \dot{\eta}_{n} \\
& =-\Delta \phi_{m}^{(k)} u_{k} c_{\mathrm{MAX}} \sum_{n=1}^{N_{m}} \Delta \phi_{n}^{(k)} \dot{\eta}_{n} \\
& =-\Delta \phi_{m}^{(k)} u_{k} c_{\mathrm{MAX}} \mathrm{L}_{k} \Phi \dot{\eta} \\
& =-\Delta \phi_{m}^{(k)} \underbrace{u_{k} c_{\mathrm{MAX}} \Delta \dot{q}_{k}}_{-M_{b k}},
\end{aligned}
$$

where $M_{b k}$ is the current value of the bending moment transmitted by the lockable joint.

\subsection{Energy transfer between vibrational modes}

The total energy of a vibrating structure is described by the following well-known equations

$$
\begin{aligned}
V & =\frac{1}{2}\left(\dot{\mathrm{q}}^{\mathrm{T}} \mathrm{M \dot { \textrm {q } }}+\mathrm{qKq}\right) \\
& =\frac{1}{2}(\dot{\eta}^{\mathrm{T}} \underbrace{\Phi^{\mathrm{T}} \mathrm{M} \Phi}_{\mathrm{I}} \dot{\eta}+\eta^{\mathrm{T}} \underbrace{\Phi^{\mathrm{T}} \mathrm{K} \Phi}_{\Omega^{2}} \eta)
\end{aligned}
$$

Matrix $\Omega^{2}$ is diagonal, so the total energy of the system is equal to the sum of the individual modal energies:

$$
V=\frac{1}{2} \sum_{m=1}^{N_{m}}\left(\dot{\eta}_{m}{ }^{2}+\omega_{m}{ }^{2} \eta_{m}{ }^{2}\right)=\frac{1}{2} \sum_{m=1}^{N_{m}} V_{m} .
$$

Differentiating the energy of the $m$ th mode one can obtain its increment rate

$$
\dot{V}_{m}=\dot{\eta}_{m}\left(\ddot{\eta}_{m}+\omega_{m}^{2} \eta_{m}\right)
$$

By substituting (8) into (13), the following formula is obtained:

$$
\begin{aligned}
\dot{V}_{m}=\dot{\eta}_{m} \sum_{i=1}^{N_{i}} \phi_{m i} f_{i} & -2 \mu_{m} \omega_{m} \dot{\eta}_{m}^{2} \\
& -\dot{\eta}_{m} \sum_{k=1}^{N_{k}} \sum_{n=1}^{N_{m}} u_{k} \gamma_{m n}^{(k)} \dot{\eta}_{n} .
\end{aligned}
$$

From eqn. (14) it is clear that the change of energy associated with the $m$ th mode is a combination of three constituents: the work of the external loads, the energy dissipated by the material, and the remaining energy transferred from other modes through locked joints (modal coupling effect). The energy transfer can be described as follows:

$$
\dot{\hat{V}}_{m}=-\dot{\eta}_{m} \sum_{k=1}^{N_{k}} \sum_{n=1}^{N_{m}} u_{k} \gamma_{m n}^{(k)} \dot{\eta}_{n}=\sum_{k=1}^{N_{k}} \sum_{n=1}^{N_{m}} \dot{V}_{m n}^{(k)}
$$

or in matrix notation

$$
\dot{\hat{V}}_{m}=-\dot{\eta}_{m} \sum_{k=1}^{N_{k}} u_{k} \Gamma_{k, m \text {-th row }} \dot{\eta} \text {. }
$$

From the equation (10) it follows that the energy transfer $\dot{\hat{V}}_{m}$ in the physical interpretation is a power related to the mechanical work done by the modal force $f_{m k}$ on a modal displacement $\eta_{m}$. Thus equation (16) can be expressed in the form of the equation below:

$$
\begin{aligned}
\dot{\hat{V}}_{m} & =\dot{\eta}_{m} \sum_{k=1}^{N_{k}} f_{m k} \\
& =\dot{\eta}_{m} \Delta \phi_{m}^{(k)} \sum_{k=1}^{N_{k}} M_{b k}
\end{aligned}
$$

The sum over indices $k$ denotes that the total instantaneous energy transfer $\dot{\hat{V}}_{m}$ is equal to sum of particular transfers through each $k$ th joint, which provides the modal force $f_{m k}$.

\section{THE PROPOSED SEMI-ACTIVE CONTROL STRATEGY}

According to eqn. (12) the energy of the $m$ th mode can increase only at the expense of the energy of the remaining modes. Let us denote by $p$ the index of the target mode to which the energy is going to be transferred. Then, using eqns (12) and (16), the $p$ th mode energy can be maximized by proper switching of the lockable joints distributed across the structure. Such a control strategy assures that the system cannot be destabilized, since no new energy is introduced during the control process [14]. 


\subsection{Maximization of instantaneous energy transfer}

As it was mentioned earlier, to transfer energy to the target mode, the derivative of its energy with respect to time has to be maximized. Mathematically, it can be written as follows:

$$
\mathrm{u}^{*}=\arg \max _{\mathrm{u} \in U} \dot{\hat{V}}_{p}
$$

where $\mathrm{u}=\left[\begin{array}{lllll}u_{1} & \cdots & u_{k} & \cdots & u_{N_{k}}\end{array}\right]^{\mathrm{T}}$ is a vector of the control signals, and $U=\{0,1\}^{N_{k}}$ is a set of the allowable control signals. A control law that satisfies the above requirement is not globally optimal in the variational sense, as for example a control law derived from Pontryagin's maximum principle. The control law derived from eqn. (18) is optimal only at a given time instance, hence it can be called instantaneous optimal control. By substituting (16) into (18), the following relation is obtained:

$$
\mathrm{u}^{*}=\arg \min _{\mathrm{u} \in U} \dot{\eta}_{p} \sum_{k=1}^{N_{k}} u_{k} \Gamma_{k, p \text {-th row } \dot{\eta}}
$$

From eqn. (19) it can be seen that it is advantageous to lock the $k$ th controllable joint at the time instant, at which

$$
\dot{\eta}_{p} \Gamma_{k, p \text {-th row }} \dot{\eta}<0 .
$$

The condition above is based on the simple rule: lock if the sign of the energy transfer to the targeted vibration mode trough the $k$ th joint is profitable, and unlock when the value of the energy transfer through the joint changes its sign to the unprofitable value.

However, the condition (20) relates to the viscous model of the lockable joint only. The dependence on the full vector of the modal velocities $\dot{\eta}$ follows from near-zero relative angular velocity $\Delta \dot{q}_{k}$ of DOFs at $k$ th joint. In a friction-type connection below certain level of bending moments the relative angular velocity between joined beams vanishes: $\Delta \dot{q}_{k}=0$. Then the expression of the generalised modal force $f_{p k}$ in terms of the difference of the angular velocities $\Delta \dot{q}_{k}$ as in eqn. (10) is no longer possible. Hence, when the joint is locked the sign of the energy transfer should be measured independently of $\Delta \dot{q}_{k}$. It can be provided by measuring the bending moment $M_{b k}$ using, e.g., a pair of strain gauges in the neighbourhood of the lockable joint.

Taking into account the above considerations, the control law cannot be formulated in the form of a single formula. When the joint is unlocked, the bending moments $M_{b k}$ take unknown near-zero values. At the other hand, when the $k$ th joint is locked, the measured values of the bending moments $M_{b k}$ are required to decide when it should be unlocked. Therefore, the control law for each $k$ th joint, including modal velocities and bending moments, is proposed in the form of Algorithm 1.

\section{Algorithm 1}

1. Estimate the vector of modal coordinates $\dot{\eta}_{M}$;

2. IF $u_{k}==0$ AND $\dot{\eta}_{M p} \Gamma_{k, p \text {-th row }}^{M} \dot{\boldsymbol{\eta}}_{M}<0$ THEN $u_{k}:=1$;

3. IF $u_{k}==1$ AND $\dot{\eta}_{M p} \Delta \phi_{m}^{(k)} M_{M b k}<0$

THEN $u_{k}:=0$;

4. Go to 1.;

The vector $\dot{\eta}_{M}$ is the vector of the estimated modal velocities obtained by modal filtering (see the next subsection) and has its dimension reduced to first several modes. The matrix $\Gamma_{k}^{M}$ is the submatrix of $\Gamma_{k}$ that provides compatible dimensions with the dimension of the vector $\dot{\eta}_{M}$.

In the second line of the control algorithm, a condition analogous to (20) is checked if the joint is currently unlocked. If the condition with modal velocities is fulfilled, the structure moves in such a manner that an immediate lock of the joint is profitable.

In the third step, the sign of the current energy transfer is checked by using the measured value of the bending moment $M_{M b k}$. If the sign of the energy transfer through the currently locked joint becomes negative, then the joint is to be immediately unlocked.

The measured vector $\dot{\eta}_{M}$ contains modal velocities related to the low frequency modes only. It means that for the currently locked $k$ th joint, the term $\dot{\eta}_{M p} \Gamma_{k, p \text {-th row }}^{M} \dot{\eta}_{M}$ in Algorithm 1 can have a significantly disturbed value compared to the fullmodel based condition (20), even in a simulation including viscous model of the lockable joint, see eqns. (1)-(3). The reason is a large value of the coefficient $c_{\mathrm{MAX}}$ involved in both $\Gamma_{k}$ and $\Gamma_{k}^{M}$ matrices, see eqn. (9).

Details about measurement of quantities required by Algorithm 1 are described in the next subsection.

\subsection{Measurement and modal filtering}

Locking a controllable joint can induce vibrations in high-frequency modes, which are usually not easily measurable because of the higher number of sensors required for this purpose. Another reason is their higher frequency of oscillations: they contribute to structural motion for very short time because of their high natural damping. Nevertheless, for a finite number of displacement or velocity sensors, they can disturb estimation of the measured low-frequency modes. This phenomenon is called measurements spillover effect. The measurements spillover can cause high-frequency locking and unlocking of the joints. This phenomenon, called chattering, is impractical and disadvantageous, since it can cause fatigue problems. The simplest way to prevent it is to filter the measurement signals in such a way that they contain information 
about low-frequency modes only. This process is called modal filtering.

Modal filters have been initially investigated in the field of active control theory, a good example can be the work by Meirovitch and Baruh published in 1980 [8]. In recent years, modal filters have been intensively used for structural damage identification $[4,9]$. In short, one can say that modal filtering is a process in which selected modal coordinates and velocities are extracted from available measurement signals. In the present study, the modal filter is described by the matrix $F_{\phi}$, which is utilised in the following equation

$$
\dot{\eta}_{M}=\mathrm{F}_{\phi} \dot{\mathrm{q}}_{M},
$$

where $\dot{\mathrm{q}}_{M}$ is the measurement vector (velocity sensors in this work). The matrix $\mathrm{F}_{\phi}$ has dimensions $N_{\eta} \times N_{S}$, where $N_{\eta}$ is the number of modal velocities visible in measurement signals and $N_{S}$ is the number of the velocity sensors.

The design process for a modal filter consists of the following three steps:

1. Optimal sensor placement.

2. Selection of the weighting matrix for spatial filtering

3. Selection of the parameters for the time domain filter. This step is optional.

Step 1 Optimal sensor placement.

For the purpose of sensor placement a discretized model of the structure is utilized. From a set of many candidate sensor locations only those which maximize the determinant of the Fisher information matrix are selected. It is a combinatorial problem which has been solved with different approaches ranging from famous Effective Independence method [5] to a new approaches where combinatorial problem is replaced by a continuous one [1]. This step can be formally represented by the following optimization problem

$$
I_{S}^{*}=\arg \max _{I_{S} \subset I_{\mathrm{DOF}}} \operatorname{det}\left(\widehat{\Phi}^{\mathrm{T}} \widehat{\Phi}\right) .
$$

In eqn. (22), $I_{S}=\left\{i_{1}, \ldots, i_{N_{S}}\right\}$ denotes the set of $N_{S}$ indices of degrees of freedom selected in the optimization process. This subset is obviously much smaller than the initial set of all possible candidate locations $I_{\text {DOF }}$. Matrix $\widehat{\Phi}=\widehat{\Phi}\left(I_{S}\right)$ is obtained by removing the rows of the full modal matrix $\Phi$ at the indices that correspond to unmeasured locations and truncating it to $N_{\eta}$ first columns.

The method described in [1] is used for sensor placement. This method finds the same number of sensor locations as the number of modes to be monitored, i.e. $N_{S}=N_{\eta}$.

Step 2 Selection of the weighting matrix for spatial filtering.

The second step is based on the sensor locations selected in the first step. If $N_{S}=N_{\eta}$, then the matrix $\mathrm{F}_{\phi}$ is calculated by inverse of the matrix $\widehat{\Phi}\left(I_{S}^{*}\right)$ determined for optimal locations set:

$$
\mathrm{F}_{\phi}=\left[\widehat{\Phi}\left(I_{S}^{*}\right)\right]^{-1} \text {. }
$$

Step 3 Selection of the parameters for the timedomain filter.

If modal filter $F_{\phi}$ is insufficient to effectively reduce the measurement spillover provided by higher modes, then the time-domain filter could be selected in many different ways, for example using the Kalman approach. However, for the sake of simplicity, a simple low-pass filter is used in this paper. The low-pass filter is defined by transfer function $G(s), s \in \mathbb{C}$, described by the formula below.

$$
G(s)=\frac{1}{T s+1}=\frac{1}{\frac{1}{\omega_{\lim }} s+1}
$$

Here $T$ is the time constant, $\omega_{\text {lim }}$ is cutoff frequency [rad/s]. The gain of the low-pass filter described by the transfer function $G(s)$ is equal to 1 .

\section{NUMERICAL EXAMPLE}

Effectiveness of the proposed semi-active control is illustrated on an example of a portal framelike structure with two lockable joints (Fig. 2). All three beams have the same length and are inextensible. Vertical members are fixed in the ground. The basic geometric and material properties of the structure are shown in Table 1.

The structure has been discretized using the finite element method. Each beam has been divided into 8 finite elements. The Euler-Bernoulli beam element with cubic shape functions has been used. The first five mode shapes and the corresponding natural frequencies are shown in Fig. 3.

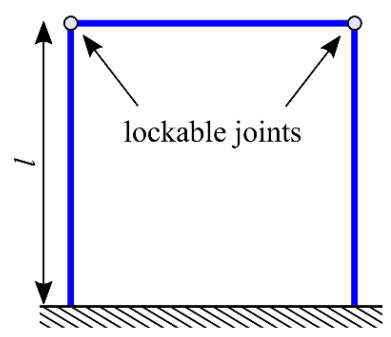

Fig. 2. Portal frame-like structure with two lockable joints

Table 1. Basic properties of the considered structure

\begin{tabular}{ccc}
\hline Beam length & $l[\mathrm{~m}]$ & 2 \\
\hline Young's modulus (steel) & $E[\mathrm{~Pa}]$ & $210 \cdot 10^{9}$ \\
\hline Material density (steel) & $\rho\left[\mathrm{kg} \mathrm{m}^{-3}\right]$ & 7860 \\
\hline Cross section area (square) & $A\left[\mathrm{~m}^{2}\right]$ & $10^{-4}$ \\
\hline Moment of inertia & $I\left[\mathrm{~m}^{4}\right]$ & $8.33^{-10}$ \\
\hline
\end{tabular}

During numerical simulation equation of motion (1) has been integrated. Each of the modes has the natural damping at $2 \%$ of the critical value. Similar models of damping are often used for steel structures. 


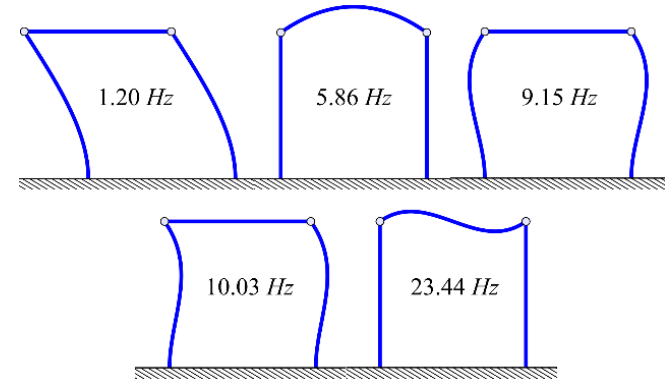

Fig. 3. First five mode shapes of the structure

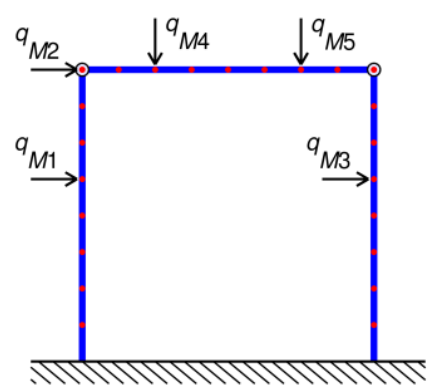

Fig. 4. Optimal placement of 5 displacement sensors

Matrix $F_{\phi}$ is designed as shown in subsection 3.2. Sensor placement is shown in Fig. 4. It has been assumed that there are five sensors which measure transverse displacements, so that the $I_{\mathrm{DOF}}$ set is limited to the translational DOFs. Red points represent the nodes of the finite element mesh. It is worth to note that sensors locations correspond with points characterised by relatively big amplitudes of motion for measured modes. Used sensor layout optimization method provides also the best effective linear independence of the monitored mode shapes at sensor locations.

The cutoff frequency of the low-pass filter $\omega_{\text {lim }}=2 \omega_{N_{\eta}}$, where $N_{\eta}=5$, that is the first five modal velocities are used to design the controller. The value of the cutoff frequency that is twice the natural frequency of the highest measured mode provides good filtering properties and simultaneously assures very small delay of filtered signals.

Measurement of the bending modes is simulated with the aid of the equation below,

$$
M_{M b k}=\mathrm{K}_{k} \mathrm{q},
$$

here $\mathrm{K}_{k}$ is the row of the stiffness matrix $\mathrm{K}$ that corresponds to the rotational DOF involved in the $k$ th lockable joint.

Three cases of modal energy transfer are shown. In the first one, the energy is transferred from the second mode to the first one. In the second case vice versa. In the third case, the energy is transferred from a combination of the second and fourth modes to the third mode. Additionally, in the third case the results are compared with the results obtained without the use of the low-pass time-domain filter.

\subsection{Case 1: Energy transfer from the second to} the first vibrational mode

In the initial condition the structure has $1 J$ of potential energy accumulated in the second mode. The first mode is selected for maximization. Results of the simulation are shown in Fig. 5, where the first five modes are plotted. Time histories of the modal energies are plotted together with sum of energies of all remaining (higher) modes $V_{H}$. Nonzero material damping caused that the total energy at the end of the simulation is around $2 / 3$ of its initial value. Energy of the targeted mode has almost a full participation in the final total energy.

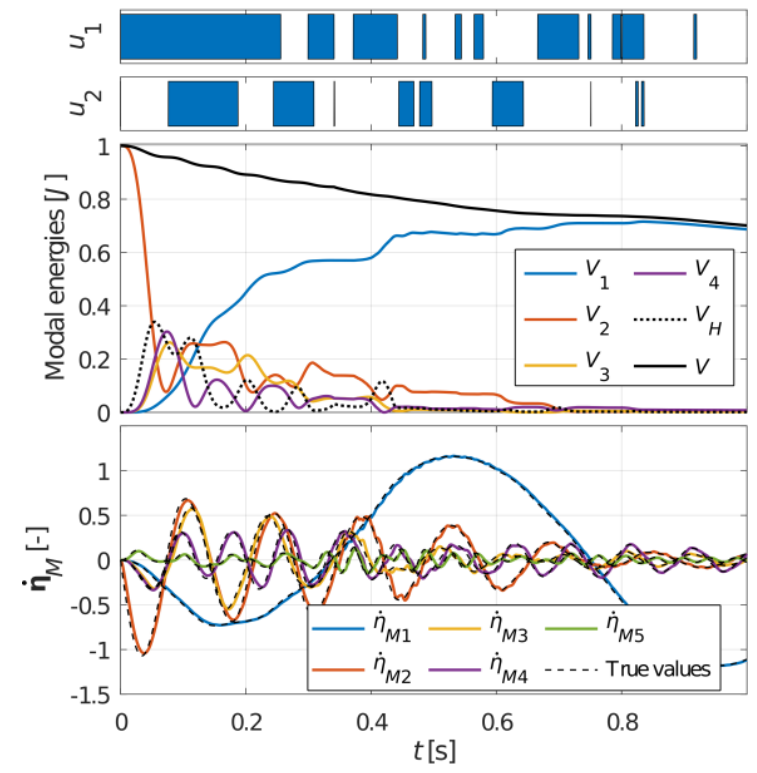

Fig. 5. Time histories of the control signals (blue colour means locked state of the joint), modal energies and measured modal velocities for case 1

As it was mentioned, the time delay of the measured modal velocities, compared with the true values calculated from the full model, is negligibly small.

Small signal disturbances caused by measurements spillover effect - especially between $0.4 \mathrm{~s}$ and $0.5 s$ are visible, but the crucial data in Algorithm 1 must be known with the accuracy of their sign. It provides a good robustness of the algorithm against the disturbances in the measurements.

The sinusoidal-like distortion visible in the time history of the first mode is a result of the modal coupling with other modes. During modal coupling, i.e., when at least one joint is currently locked, all modal coordinates and its derivatives are disturbed.

\subsection{Case 2: Energy transfer from the first to the second vibrational mode}

In this case the structure has $1 J$ of potential energy accumulated in the first mode and the second mode is selected as the target mode. Results in the form of time histories analogous as in the previous subsection are shown in Fig. 6. 
The amount of mechanical energy transferred to the selected mode is smaller than in the previous case (when the direction of the transfer is reversed). In this case locking of the joint causes the energy to be accumulated in higher modes until it is released together with the energy of the targeted mode. It is visible that when modes are released at the time instance near $0.3 \mathrm{~s}$, the total energy $V$ decreases faster because of a significant participation of higher modes in structural motion.

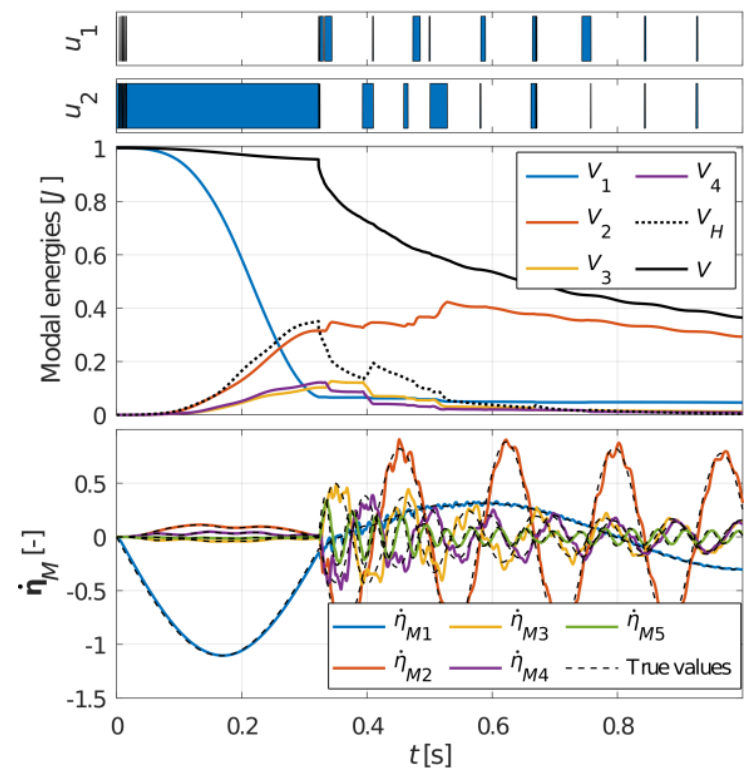

Fig. 6. Time histories of the control signals (blue colour means locked state of the joint), modal energies and measured modal velocities for case 2

The reason of the accumulation-release like behaviour is that energy is transferred from the lower order mode, which is less stiff, to the modes characterised by a larger stiffness related to the higher natural frequencies. In the time history of the measured modal velocities it is visible that before the time instant of the release, second and higher modal velocities are relatively small. It means that the majority of the kinetic energy of the first mode has been accumulated as the strain energy of the remaining modes. The first mode has a longer period than the more stiff modes, so the first mode coupled with the higher ones is stopped by them before the end of the first mode period. When the motion of the structure almost vanishes and the energy transfer between modes no longer occurs, the control algorithm unlocks the second joint at the time instant near to $0.3 \mathrm{~s}$. Undesired accumulation of the energy in the higher modes stays in good agreement with the results obtained in $[10,15]$.

After the release of the higher order modes, small disturbances of the measured modal velocities can be also observed. This effect is not visible before the release of potential energy described above, because of the quasi-static motion of these modes. Then the energy of the higher modes $V_{H}$ is potential and relates to the modal displacements which have significantly lower level compared to the velocities in free vibration, which are proportional to the natural frequencies.

\subsection{Case 3: Energy transfer from the second and fourth modes to the third mode}

In this case, at the beginning of the control process the structure accumulates $1 / 2 J$ of potential energy in the second mode and $1 / 2 J$ in the fourth mode ( $1 J$ of the total energy). The third mode is selected for maximization. Results of the simulation are presented in Fig. 7. Analogously to the previous cases, only the first five modes are shown for clarity of presentation.

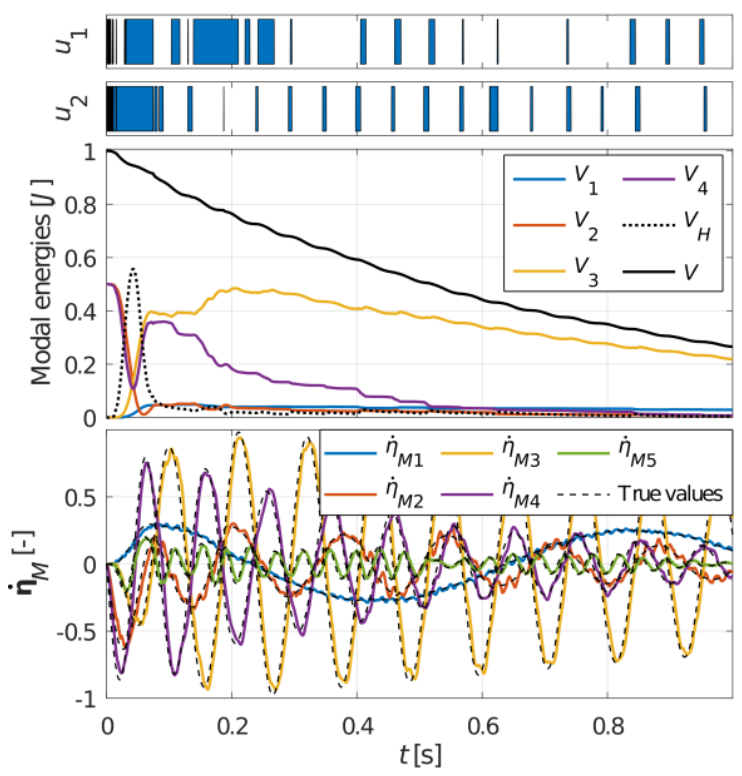

Fig. 7. Time histories of the control signals (blue colour means locked state of the joint), modal energies and measured modal velocities for case 3

This example shows the energy transfer from a combination of two modes into one selected mode. Overall material damping seems to remain constant because the natural frequency of the targeted mode has its value between the values of the initially induced modes. After $0.2 s$, the energy of the targeted mode becomes the most pronounced and it is further slowly dissipated, but its decrease is slower than that of the total energy. From the beginning up to the time instant of $0.2 \mathrm{~s}$, visible distortions of the modal coordinates appear. This effect is induced by the modal coupling caused by the locked state of the controllable joints.

Small time delays of modal velocities of the third and fourth modes are well visible in Fig. 7. Because of the higher natural frequencies of these modes, the influence of the low-pass filter is more pronounced. However, the disturbance provided by the low-pass filter is significantly smaller than the one provided by measurement spillover. Both types of disturbances of modal velocity time histories have a negligibly small influence on the control. 
Simulation for case 3 has been performed also without using the low-pass filter: spatial-based modal filtering is performed only. Results are shown in Fig. 8. Level of the mechanical energy of the targeted mode as well as level of the vibrational total energy is lower when low-pass filter is not used. The reason is the faster energy dissipation caused by the chattering of the lockable joints due to the measurement spillover effect (from 0 to 0.12 $s)$. Explanation of this phenomenon is as follows. Any locking/unlocking of the joints can excite higher vibration modes for a short time moment. These modes provide measurement spillover and can cause again an incorrect locking or unlocking in future time instances. It results in an amplification of this effect (see the dashed box in Fig. 8) and the chattering of the lockable joints.

It should be noted that even with strong disturbance of the measured modal velocities $\dot{\eta}_{M}$ and chattering occurring in simulations, performance of the control is not degraded. Only a faster energy dissipation in the joints and through material damping of the higher order modes occurs.

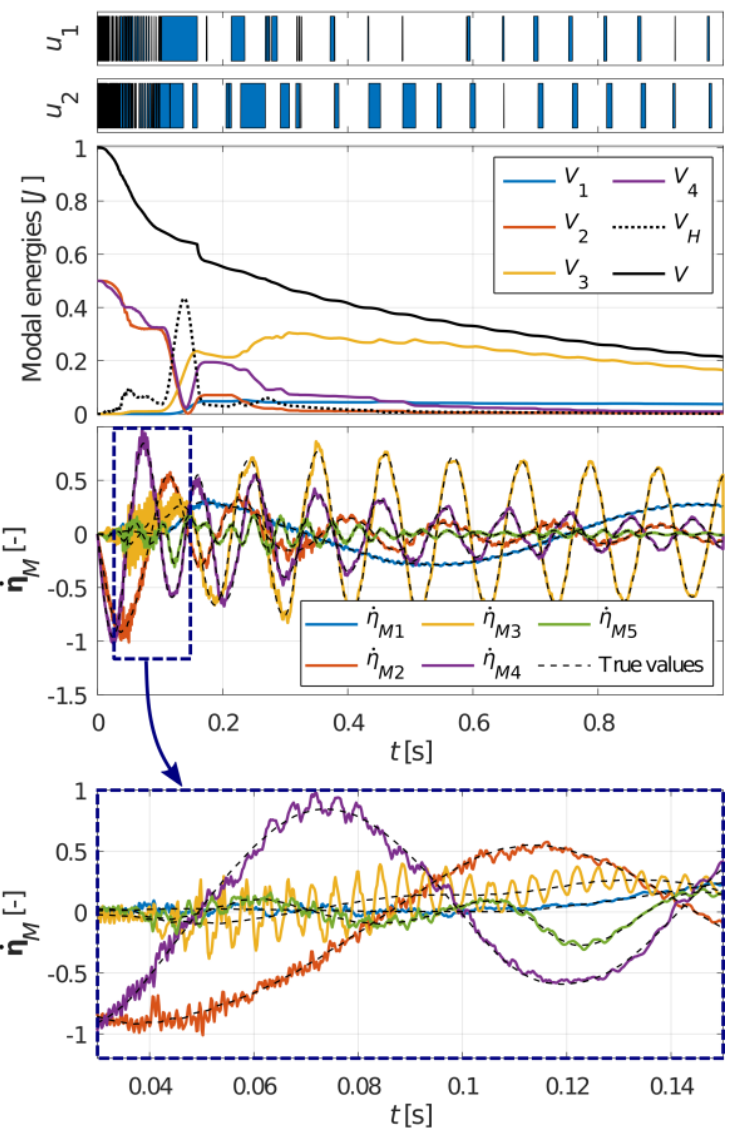

Fig. 8. Time histories of the control signals (blue colour means locked state of the joint), modal energies and measured modal velocities for case 3 without using the low-pass filter

\section{CONCLUSIONS}

It has been shown that instantaneous maximization of energy transfer to the targeted mode can be used for a directed modal energy transfer. The control law is developed to be implemented in a specific type of semi-active elements in the form of lockable structural joints. Thanks to the semi-active form of the proposed control, the system is always stable and needs no high power supply.

Numerical results show that the energy transfer can be performed even when only a few lowfrequency modes and bending moments near to the lockable joints are estimated. However to obtain a good performance of the overall control system, the mathematical model of the structure should be analysed first in order to find the optimal sensor placement and to design the modal filter, which is a part of the control law. The control algorithm can be called robust against measurement spillover. Moreover, its performance is not degraded for significant disturbances, even if they result in joint chattering. The obtained results show that the transfer from higher-order modes to the lower modes is more efficient than vice versa. The reason is the difference in stiffnesses of modes.

A further research challenge is the problem of optimum placement of a limited number of lockable joints in a large structure for the purpose of efficient modal energy transfer [16].

\section{ACKNOWLEDGEMENT}

The authors gratefully acknowledge the support of the National Science Centre, Poland, granted under grant agreements 2018/31/B/ST8/03152, 2017/26/D/ST8/00883 and 2017/25/B/ST8/01800.

\section{REFERENCES}

1. Błachowski B, Świercz A, Ostrowski M, Tauzowski $\mathrm{P}$, Olaszek P, Jankowski Ł. Convex relaxation for efficient sensor layout optimization in large scale structures subjected to moving loads. ComputerAided Civil and Infrastructure Engineering 2020; in review.

2. Casciati F, Magonette G, Marazzi F. Technology of Semi-active Devices and Applications in Vibration Mitigation. United Kingdom, Chichester: John Wiley \& Sons, Ltd; 2006. https://doi.org/10.1002/0470022914

3. Cassidy IL, Scruggs JT, Behrens S, Gavin HP. Design and experimental characterization of an electromagnetic transducer for large-scale vibratory energy harvesting applications. Journal of Intelligent Material Systems and Structures. 2011; 22(17): 2009-2024. https://doi.org/10.1177/1045389X11421824

4. Dworakowski Z, Ambroziński $\mathrm{L}$, Mendrok K. Damage localization in plates with use of the procedure based on Modal Filtration. 11th International Conference on Damage Assessment of Structures (DAMAS 2015). Journal of Physics Conference Series. 2015; 628: 012028. August 24-26, 
2015. Ghent, Belgium.

https://dx.doi.org/10.1088/1742-6596/628/1/012028

5. Kammer DC. Sensor Placement for On-Orbit Modal Identification and Correlation of Large Space Structures. Journal of Guidance, Control, and Dynamics. 1991; 14:251-259. https://doi.org/10.2514/3.20635

6. Kęcik K. Assessment of energy harvesting and vibration mitigation of a pendulum dynamic absorber. Mechanical Systems and Signal Processing. 2018; 106:198-209. https://doi.org/10.1016/j.ymssp.2017.12.028

7. Lu L. Semi-active modal control for seismic structures with variable friction dampers. Engineering Structures. 2004;26(4):437-454. https://doi.org/10.1016/j.engstruct.2003.10.012

8. Meirovitch L. Baruh H. Control of self-adjoint distributed-parameter systems, Journal of Guidance, Control and Dynamics. 1982;(1):60-66. https://doi.org/10.2514/6.1980-1707

9. Mendrok K, Wojcicki J, Uhl T. An application of operational deflection shapes and spatial filtration for damage detection. Smart Structures and Systems 2015; 16(6): 1049-1068. https://dx.doi.org/10.12989/sss.2015.16.6.1049

10. Mróz A, Holnicki-Szulc J, Biczyk J. Prestress accumulation-release technique for damping of impact-born vibrations: application to self-deployable structures. Mathematical Problems in Engineering 2015: 720236. http://dx.doi.org/10.1155/2015/720236

11. Ostrowski M, Błachowski B, Jankowski Ł, Pisarski D. Semi-active control of mechanical energy transfer between vibrational modes. Book of abstracts of SOLMECH 2018 - 41st Solid Mechanics Conference. August 27-31, 2018. Warsaw, Poland.

12. Ostrowski M, Błachowski B, Jankowski Ł, Pisarski D. Inverse Lyapunov based method for semi-active control of energy transfer between vibrational modes. Conference proceedings of SMART 2019 - IX ECCOMAS Thematic Conference on Smart Structures and Materials. July 8-11, 2019. Paris, France.

13. Park Y, Kim K. Semi-active vibration control of space truss structures by friction damper for maximization of modal damping ratio. Journal of Sound and Vibration 2013;332(20):4817-4828. https://doi.org/10.1016/j.jsv.2013.04.032

14. Pisarski D. Decentralized stabilization of semi-active vibrating structures. Mechanical Systems and Signal Processing. 2018;100:694-705. https://doi.org/10.1016/j.ymssp.2017.08.003

15. Popławski B, Mikułowski G, Mróz A, Jankowski Ł. Decentralized semi-active damping of free structural vibrations by means of structural joints with an on/off ability to transmit moments. Mechanical Systems and Signal Processing 2018; 100: 926-939.

https://doi.org/10.1016/j.ymssp.2017.08.012

16. Popławski B, Mikułowski G, Pisarski D, Wiszowaty R, Jankowski Ł. Optimum actuator placement for damping of vibrations using the PrestressAccumulation Release control approach. Smart Structures and Systems. 2019;24(1):27-35. https://doi.org/10.12989/sss.2019.24.1.027

17. Wei C, Jing X. A comprehensive review on vibration energy harvesting: Modelling and realization. Renewable and Sustainable Energy Reviews 2017; 74: 1-18. https://doi.org/10.1016/j.rser.2017.01.073
Received 2019-10-29

Accepted 2020-01-20

Available online 2020-01-21

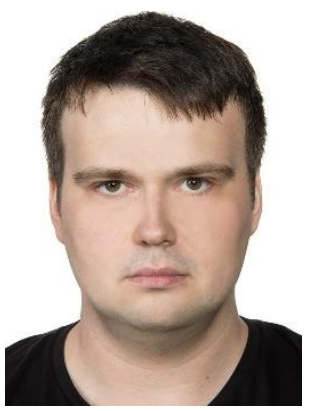

Mariusz OSTROWSKI is PhD Student at the Institute of Fundamental Technological Research of the Polish Academy (IPPT PAN) of Sciences in Warsaw (Poland) since 2017. $\mathrm{He}$ received the Master degree at Lublin University of Technology in 2016. He worked for space industry (UV2VIS project, which is related with Sentinel-5 mission) from 2017 to 2018. His current research covers the fields of semi-active modal control of vibration and optimal sensors placement. His main interests are: model-based and optimal control, mathematical modelling, optimisation.

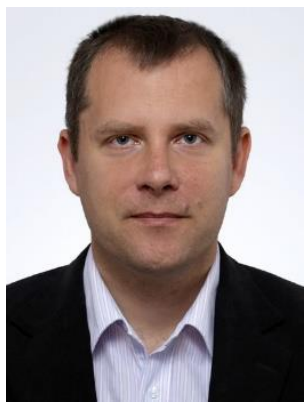

Bartlomiej BLACHOWSKI is a member of the scientific staff of the Institute of Fundamental Technological Research of the Polish Academy of Sciences (IPPT PAN). He received the MSc degree at the Silesian University of Technology in 2000 and the $\mathrm{PhD}$ degree at IPPT PAN in 2005. His research areas span wide-sense structural dynamics, in particular strategies of vibration control, algorithms for optimal structural design, and the use of sensor networks for structural health monitoring. $\mathrm{He}$ is equally interested in modern computational methods of structural analysis, experimental techniques such as operational modal analysis, and hardware solutions for wireless acceleration measurements of engineering structures.

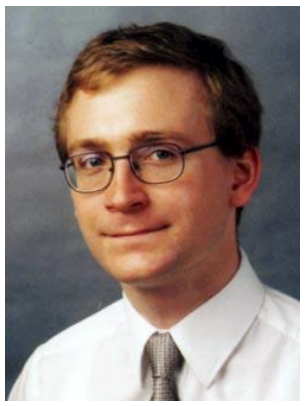

Lukasz JANKOWSKI is an associate professor at the Institute of Fundamental Technological Research of the Polish Academy of Sciences (IPPT PAN). He received the MSc degrees in mathematics (2001) and in computer science (2000), the $\mathrm{PhD}$ degree in mathematical physics (2004), and the DSc in mechanics (IPPT PAN, 2014). The research interests involve the area of inverse problems in structural mechanics and include smart, adaptive structures, semi-active structural control and structural health monitoring.

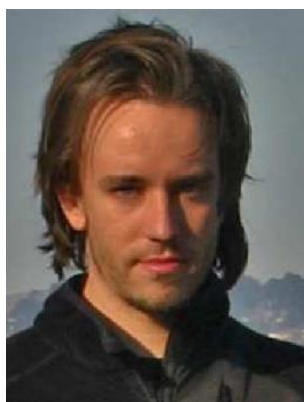

Dominik PISARSKI received the M.Eng. degree in automatic control from AGH University, Cracow, Poland, in 2006. In 2012 he received the Ph.D. degree in mechanics from Institute of Fundamental Technological Research, Polish Academy of Sciences, Warsaw, Poland. In 2014 he received the Ph.D. degree in automatic control in the Department of 
Automatic Control, Grenoble University, France. Since 2014 he has been working as an Associate Professor in Department of Intelligent Technologies in Institute of Fundamental Technological Research, Polish Academy of Sciences, Warsaw, Poland. His research interest focuses on control and optimization of complex mechanical and transportation systems. $\mathrm{He}$ is an author of over 50 publications, including 21 papers in international journals, 23 international conference papers, 1 book and 2 book chapters. 